\title{
Docetaxel HIPEC Combined with Endostar versus Docetaxel HIPEC Alone in Treatment of Malignant Ascites $\llbracket A$ Retrospective Study
}

Jing Wu

Zhejiang Hospital

Hao Jiang

Zhejiang Hospital

Liting Chen

Zhejiang Hospital

Bingqi Yu

Zhejiang Hospital

Zhibing Wu ( $\nabla$ wuzhibingzjyy@163.com )

Zhejiang Hospital https://orcid.org/0000-0003-3016-9264

\section{Research}

Keywords: Docetaxel, Hyperthermic intraperitoneal chemotherapy (HIPEC), Recombinant human endostatin/Endostar, Malignant ascites

Posted Date: March 22nd, 2021

DOI: https://doi.org/10.21203/rs.3.rs-306501/v1

License: (c) (i) This work is licensed under a Creative Commons Attribution 4.0 International License. Read Full License 


\section{Abstract}

Objective区The purpose of this study is to retrospectively observe the clinical efficacy and adverse reactions of docetaxel hyperthermic intraperitoneal chemotherapy (HIPEC) combined with endostar in treatment of malignant ascites.

Methods $\$ 56$ cases of malignant ascites admitted to Zhejiang Hospital from July 2019 and October 2020 had received no less than second-line chemotherapy. The observation group $(n=29)$ was treated with endostar 60mg d1,4,7 Q3W intraperitoneal injection combined with docetaxel $60 \mathrm{mg} / \mathrm{m}^{2} \mathrm{~d} 4$ HIPEC Q3W for 2 cycles. The control group $(n=27)$ was just treated with docetaxel $60 \mathrm{mg} / \mathrm{m}^{2} \mathrm{~d} 1$ HIPEC Q3W for 2 cycles. The general clinical data and qualitative data of the treatment results were processed by SPSS26.0 using $\chi^{2}$ test, and quantitative data were processed by t test. When $P<0.05$, statistical data can be considered statistically significant.

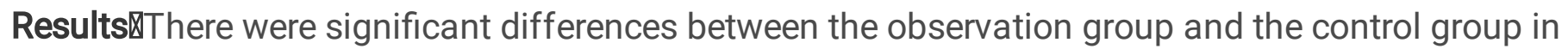
objective response rate (ORR) $(65.5 \%$ vs $37.0 \% \otimes P=0.033)$ in the improvement rate of KPS $(48.3 \%$ vs $22.2 \%, P=0.042$ ) $\$ also in the median control time ( $57 \mathrm{dvs} 45 \mathrm{~d} \otimes P=0.027)$. The incidence of III to IV adverse reactions was low, and no treatment-related death was observed. There was no significant difference in the incidence of adverse reactions between the two groups.

Conclusion区Endostar combined with docetaxel HIPEC has good clinical effect on malignant ascites and can improve patients' quality of life, and the adverse reactions can be tolerated.

\section{Introduction}

Malignant ascites (MA) is a common complication of advanced malignant tumors, which can lead to abdominal discomfort, nausea, vomiting, limb edema, dyspnea, anorexia and cachexia[1]. These symptoms greatly deteriorate the patients' quality of life. MA is common in abdominal and pelvic malignancies such as ovarian, colorectal, pancreatic, gastric, and primary peritoneal cancers, as well as extra-abdominal tumors such as lung and breast cancers. MA is often stubborn, simple drainage of ascetic can only temporarily alleviate symptoms, which will be quickly generated without effective treatment. Repeated paracentesis can lead to a number of problems, including pain, hypovolemia, protein loss, infection, peritonitis and bowel perforation[2]. Patients with MA have very poor prognoses, with a median survival of 3-6 months, and there are almost no long-term survivors. It is important to effectively reduce or even eliminate ascites, relieve the clinical symptoms of patients, and improve the quality of life.

Hyperthermic intraperitoneal chemotherapy (HIPEC) is a treatment with curative intent for peritoneal metastasis of abdominal and pelvic tumors since it was first reported by Spratt et al in 1980[3]. HIPEC has a good effect on treatment and prevention of peritoneal metastasis of gastric cancer, colorectal cancer, peritonel pseudomyoma and malignant peritoneal mesothelioma. Especially for the malignant peritoneal effusion control, HIPEC has a unique advantage[4,5]. The aim of our study was to evaluate the 
clinical efficacy and adverse reactions of docetaxel HIPEC combined with endostar in treatment of malignant ascites. Patients' QOL and ascites control time were evaluated as the outcome.

\section{Material Methods}

\section{General Information}

A total of 56 patients with advanced malignancies associated with malignant ascites were enrolled in the Department of Oncology, Zhejiang Hospital (Hangzhou, China) between July 2019 and October 2020. Inclusion Criteria: 1. Histopathological confirmation of cancer and positive cytology of ascites; 2.

Received second-line or above systemic chemotherapy, had not received docetaxel before, or had received the last dose of docetaxel more than six months ago; 3 . Adequate bone marrow function (neutrophil count $\geq 1,500 / \mathrm{ml}$, Hemoglobin $\geq 90 \mathrm{~g} / \mathrm{L}$, and platelet count $\geq 90,000 / \mathrm{ml}$ ); adequate liver function (serum total bilirubin level $<1.5$ times the upper normal limit, and serum transaminase levels $<2$ times the upper normal limit); adequate renal function (serum creatinine level $₫ 1.5 \mathrm{mg} / \mathrm{dl}$ ); 4 . The expected survival time was over 3 months. Exclusion criteria: 1.Uncontrollable brain metastasis; 2 . Severe heart and lung disease; 3.Prominent compartmental ascites; 4 . Uncontrolled infection. The patients were randomly allocated into endostar + docetaxel (observation group, $n=29$ ) and docetaxel only (control group, $n=27$ ) groups. The 56 patients consisted of $26(46.4 \%)$ males and $30(53.6 \%)$ females, with a median age of 55.5 (ranged from 26 to 78) years. There was no statistical significance in gender, age, ascites volume and KPS score between the two groups ( $P \otimes 0.05$ ) (see Table 1 for details).

\section{Treatment}

Ascites volume was estimated by B ultrasound. Bilateral abdominal paracentesis and catheterization was performed under $B$ ultrasound guidance. The ascites was drained as slowly as possible in 1 to 3 days. The ascites volume was counted and abdominal circumference was measured. The HIPEC was performed with a thermo-perfusion machine (Jilin Maida RHL-2000A). The temperature was controlled at 43 to $45^{\circ} \mathrm{C}$, and the abdominal cavity was irrigated with $1000-2000 \mathrm{ml}$ heated normal saline before the infusion of chemotherapy drugs. The observation group $(n=29)$ was treated with endostar $60 \mathrm{mg}$ d1,4,7 Q3W intraperitoneal injection combined with docetaxel $60 \mathrm{mg} / \mathrm{m}^{2} \mathrm{~d} 4$ HIPEC Q3W for 2 cycles. The control group ( $\mathrm{n}=27$ ) was just treated with docetaxel $60 \mathrm{mg} / \mathrm{m}^{2} \mathrm{~d} 1$ HIPEC Q3W for 2 cycles. Dexamethasone tablets $4 \mathrm{mg}$ bid was taken orally for 3 days before and during application of docetaxel. Patients' blood pressure, heart rate, respiration rate, and blood oxygen saturation values were monitored during the HIPEC. The clinical efficacy, side effects and prognosis of the patients were investigated.

\section{Evaluation and determination of efficacy}

The ascites control time was calculated from the day when the drainage tube was pulled out at the end of the second cycle of treatment. Abdominal circumference was measured twice a week. According to WHO standards, the patients were divided into complete remission (CR), partial remission (PR), disease stability (SD), and disease progression (PD). CR means ascites disappeared for more than 4 weeks, PR means 
ascites decreased by more than $50 \%$ and lasted more than 4 weeks, SD means ascites decreased by less than $50 \%$ or increased by less than $25 \%$, and PD means ascites increased by more than $25 \%$. Objective response rate $(\mathrm{ORR})=(\mathrm{CR}+\mathrm{PR}) /$ total number of cases $\times 100 \%$. Follow-up was terminated when cases reached $\mathrm{PD}$ or 90 days.

\section{Evaluation criteria of QOL}

According to the Kamofsky Performance Status (KPS) Scale, the changes in patients' KPS scores before and after treatment were evaluated. After treatment, if the KPS score increased/ decreased by less than 10 points and lasted for more than 4 weeks, then the patients were deemed as stable. KPS increased by $\geq 10$ points was considered as improved囚and reduced by $\geq 10$ was judged as a decline.

\section{Safety evaluation}

Adverse reactions were classified into I -IV degrees according to Common Terminology Criteria Adverse Events (CTCAE) Version 4.0 of National Cancer Institute.

\section{Statistical analysis}

The general clinical data and qualitative data of the treatment results of 56 cases were processed by SPSS version 26.0 using $X^{2}$ test and quantitative data were processed by $t$ test. The ascites control time was compared using the Kaplan-Meier method. Statistical data can be considered statistically significant when $P \llbracket 0.05$.

\section{Results}

There were no significant differences between the two groups in gender, age, KPS score, ascites volume and course of treatment before. $₫ P \otimes 0.05$,see Table 1 for details $\varangle$

\section{Response Evaluation}

The short-term efficacy was evaluated. ORR of patients in the observation group was $65.5 \%$ and ORR of patients in the control group was $37 \%$. The difference between the two groups in ORR was statistically significant $(P \otimes 0.05)$ (Table 2).

\section{QOL Evaluation}

In the observation group, the improvement rate of KPS was $48.4 \%$ after treatment. In the control group, the improvement rate of KPS is $22.2 \%$ after treatment. There was significant difference in the improvement rate of KPS between the two groups $₫ P=0.042 \bigotimes$.(Table 3 ).

\section{Ascites control time}


In the observation group, the median ascites control time was $57 \mathrm{~d}(10 \otimes 90 \mathrm{~d})$, and in the control group the median ascites control time was $45 \mathrm{~d}(7 \rrbracket 85 \mathrm{~d})$. There was significant difference between the two groups ( $P$ $=0.027)$. (Fig 1)

\section{Adverse Reactions}

Both groups had varying degrees of leukopenia, anemia, thrombocytopenia, nausea and vomiting, decreased appetite, constipation, abdominal pain and diarrhea. There were no significant differences in the incidence of the adverse reactions between the two groups of patients $(P \otimes 0.05)$. The incidence of III to IV adverse events was low, and no treatment-related death was observed. No serious complications such as intestinal obstruction, peritonitis, gastrointestinal bleeding and abdominal infection occurred in both groups. There was no hypohepatia of grade III to IV in the two group. In the observation group, 3 cases had I to II palpitation and chest tightness, and 5 cases had I to II elevation of blood pressure, which were relieved after symptomatic treatment, and the follow-up therapy was not affected.

\section{Discussion}

The pathophysiology of MA is thought to be a combination of obstructed lymphatic drainage and altered vascular permeability[1]. Vascular endothelial growth factor (VEGF) expressed by the primary tumor plays an important role in the pathophysiological mechanism of ascites formation. VEGF is a major contributor to tumor neovascularization and increased capillary permeability, which in turn contribute to MA accumulation[6, 7]. Tumors for example ovarian, gastric, colon, pancreatic cancer, and peritoneal metastases, associated with increased concentrations of VEGF[8]. The VEGF value in malignant ascites was significantly higher than that in benign ascites, and the higher the VEGF value, the worse the prognosis of patients. In view of the important pathogenetic role of VEGF, one of the strategies is VEGF inhibition with the aim to reduce MA formation[9].

Recombinant human endostatin injection/Endostar is an anti-tumor angiogenesis targeting drug developed in China, which was approved in 2005 by the State Food and Drug Administration of China (SFDA) for the treatment of non small cell lung cancer (NSCLC).Afterwards it is used to treat different types of tumors combined with chemotherapy in clinical practice[10]. An animal experiment showed that the possible mechanisms of endostar controlling malignant ascites were to reduce VEGF secretion and circulating endothelial cells(CECs) in peripheral blood, decrease the permeability of the peritoneum and tumor neovascularization, reduce the leakage of erythrocytes into the ascites and lower the content of MMP-2, osteopontin and integrin in mouse ascites and serum[9, 11].

In recent years HIPEC becomes an effective auxiliary method for the treatment of malignant tumors in the abdominal cavity. First the infusion fluid can mechanically remove abdominal isolated cancer cells. Then during the process, chemotherapeutic drugs are infused into the abdominal cavity, so as to form a circumstance of high and durable concentration in the peritoneal cavity[12]. There is a natural peritonealplasma barrier (PPB) in our body,which limits the resorption of drugs from the peritoneal cavity into the blood[13]. The sequestration of chemotherapeutic agents especially high molecular drugs improves the 
abdominal cytotoxicity and reduces systemic toxicity. The hydrophilic of chemotherapy agents and the macromolecular of the drugs can prolong retention time in the peritoneal cavity[14]. Furthermorelthe choice of drugs under hyperthermic conditions is important because suitable hyperthermia can accelerate velocity of drug permeability into tumor tissue. So optimization of therapy regimen can increase cytotoxicity at the peritoneal lesions without an increase in systemic (especially bone marrow) toxicity[ 15 , 16].

Docetaxel is a semisynthetic taxane, which is widely used for NSCLC, breast cancer,gastric cancer,ovarian cancer,et al[17]. It showed nearly 1000 times higher area under the curve(AUC) with intraperitoneal injection compared with intravenous injection for peritoneal concentrations[18]. Also the peak concentration was approximately 200 times higher in the peritoneum than in the plasma[19, 20].

So how is the effect of docetaxel combined with endostar in treating MA匹Our research adopted docetaxel as the hyperthermic intraperitoneal chemotherapy agent, combined with endostar or not to treat with MA patients. There were significant differences between the observation group and the control group in ORR( $65.5 \%$ vs $37.0 \%$ ), in the improvement rate of KPS (48.3\% vs $22.2 \%$ ) पand in the median control time (57d vs 45d).Also the incidence of III to IV adverse reactions was low, and no treatment-related death was observed. It indicated that the combination of these two drugs had good tolerance.

At the end of the 90-day follow-up, there were still 2 patients assessed as CR in the observation group, and both had ovarian cancer, which may indicate a better efficacy for MA patients with ovarian cancer. For further confirmation, a subgroup analysis or a more rational prospective clinical study is needed. In addition, we found that 5 patients with serum creatinine level approach to $1.5 \mathrm{mg} / \mathrm{dl}$ were detected with varying levels of decreased creatinine after treatment. More clinical observations are needed to verify whether this therapy has a protective effect on renal function.

The multicenter, randomized controlled study of Qin S showed a synergistic effect with endostar and cisplatin in improving the clinical efficacy, TTP (time to disease progression) and QOL in treatment of malignant hydrothorax and ascites. The median TTP was $69 \mathrm{~d}$ and the ORR was $63 \%[21]$. In our study, the ORR (65.5\%) of the observation group was similar to Qin S's. The median time of ascites control (57d) was less than Qin S's, considering the fact that all patients enrolled in our study were treated with secondline or above treatments, the sample size was small, and the retrospective analysis might have certain bias, etc.

Currently for MA patients with different kinds of tumors, there is no clear guidelines to standardize medication at the moment[22]. It is absolutely accepted that the combination treatment of antiangiogenesis drugs and different chemotherapy drugs may have different impact on curative effect. It is unknown whether the effect is synergistic, additive, or antagonistic. So relevant prospective clinical trials need to be designed for further observational studies. Continued optimal utilization of intraperitoneal chemotherapy agents in the HIPEC in MA is desirable. 


\section{Conclusion}

In brief, docetaxel hyperthermic intraperitoneal chemotherapy combined with endostar can significantly control malignant ascites and has relatively mild adverse reactions, which is worthy of clinical application and promotion.

\section{Declarations}

\section{Ethics approval and consent to participate}

The study was done after an agreement from Zhejiang Hospital ethics committee and with the patients' informed consent.

\section{Consent for publication}

Not applicable.

\section{Availability of data and materials}

The data generated or analyzed during this study are available from the corresponding author on reasonable request.

\section{Competing interests}

The authors declare that they have no competing interests.

\section{Funding}

Not applicable.

\section{Authors' contributions}

Data collection and analysis were performed by Jing Wu, Liting Chen and Bingqi Yu. Jing Wu and Hao Jiang contributed to the literature study analysis for discussion and participated in treatment procedures. Zhibing Wu is the corresponding author of this article. All authors read and approved the final manuscript in its present form.

\section{Acknowledgements}

Not applicable.

\section{Authors' information}

All the uthors come from Department of Oncology, Zhejiang Hospital, Hangzhou, Zhejiang, China. 


\section{References}

1. Cavazzoni E, Bugiantella W, Graziosi L, et al. Malignant ascites: pathophysiology and treatment. Int J Clin Oncol.2013;18(1):1-

2. Becker G, Galandi D, Blum HE. Malignant ascites: systematic review and guideline for treatment. Eur J Cancer,2006,42(5):589-597

3. David L, Bartlett MD. HIPEC: the complexities of clinical trials. Ann Surg Oncol,2008,15(5):1277-1279

4. Cortes-Guiral D, Glehen O. Expanding Uses of HIPEC for Locally Advanced Colorectal Cancer: A European Perspective. Clin Colon Rectal Surg,2020,33(5):253-257

5. Rau B, Brandl A, Thuss-Patience P,et al. The efficacy of treatment options for patients with gastric cancer and peritoneal metastasis. Gastric Cancer,2019

6. Gamblin V, Da SilvaA, Villet S,et al. Supportive care for malignant ascites in palliative phase: Place of paracentesis and diuretics. Bulletin du Cancer,2015,102(11): 940-945

7. Zhan N, Dong WG, Wang J. The clinical significance of vascular endothelial growth factor in malignant ascites. Tumour Biol,2016, 37(3): 3719-3725

8. Becker G, Blum HE. VEGF Trap for the treatment of malignant ascites.Lancet Oncol, 2012,13(2)凶115116

9. Wei H, Qin S,Yin X,et al. Endostar inhibits ascites for matiom and prolongs survival in mouse models of malignant ascites.Oncol lett,2015,9(6):2694-2700

10. Jiang Z, Qin S. Study progression of recombinant human endostatin (Endostar) for the treatment of malignant serous effusion. Chinese-German J Clin Oncol,2011,10: 435-441

11. Chao J, Qin S. Progression of Recombination Human Endostatin (Endostar) Intraperitoneal Injection for the Treatment of malignant ascites. Chinese Clinical Oncology, 2019,24(9):856-861

12. Jiao J, Li C, Yu G,et al. Efficacy of hyperthermic intraperitoneal chemotherapy (HIPEC) in the management of malignant ascites. World J Surg Oncol,2020,18(1):180

13. Jacquet P, Sugarbaker PH. Peritoneal-plasma barrier. Cancer Treat Res,1996,82:53-63

14. Goodman MD, McPartland S, Detelich D,et al. Chemotherapy for intraperitoneal use: a review of hyperthermic intraperitoneal chemotherapy and early post-operative intraperitoneal chemotherapy. J Gastrointest Oncol, 2016,7(1):45-57

15. Moussa M, Goldberg SN, Kumar G, Levchenko T, Torchilin V, Ahmed M. Effect of thermal dose on heat shock protein expression after radio-frequency ablation with and without adjuvant nanoparticle chemotherapies. Int J Hyperthermia. 2016,32(8):829-

16. Pletcher E, Gleeson E, Labow D. Peritoneal Cancers and Hyperthermic Intraperitoneal Chemotherapy. Surg Clin North Am,2020,100(3):589-613

17. Koemans WJ, van der Kaaij RT, Wassenaar ECE, et al. Systemic exposure of oxaliplatin and docetaxel in gastric cancer patients with peritonitis carcinomatosis treated with intraperitoneal hyperthermic chemotherapy. Eur J Surg Oncol,2020 
18. Tsubamoto H, Takeuchi S, Ito K, Miyagi Y, et al. Feasibility and efficacy of intraperitoneal docetaxel administration as salvage chemotherapy for malignant gynaecological ascites. J Obstet Gynaecol,2015,35(1):69-73

19. Morgan RJ Jr, Doroshow JH, Synold T, et al. Phase I trial of intraperitoneal docetaxel in the treatment of advanced malignancies primarily confined to the peritoneal cavity: dose-limiting toxicity and pharmacokinetics. Clinical Cancer Research, 2003,9:5896-5901.

20. Marchettini P, Stuart OA, Mohamed F, et al. Docetaxel: pharmacokinetics and tissue levels after intraperitoneal and intravenous administration in a rat model. Cancer Chemother Pharmacol,2002,49(6):499-503

21. Qin S, Yang L, Liang J, et al. Intra-pleural injection of recombinant human endostatin and/or cisplatin in treatment of malignant hydrothorax and ascites: A multicenter randomized controlled trial. Chinese Clinical Oncology, 2017,22(3):193-202

22. Li JX, Shi YM, An LY, et al. Quality assessment of the guidelines for the management of malignant pleural effusions and ascites. World J Surg Oncol,2020,18(1):331

\section{Tables}

Due to technical limitations, table $1,2,3,4$ is only available as a download in the Supplemental Files section.

\section{Figures}




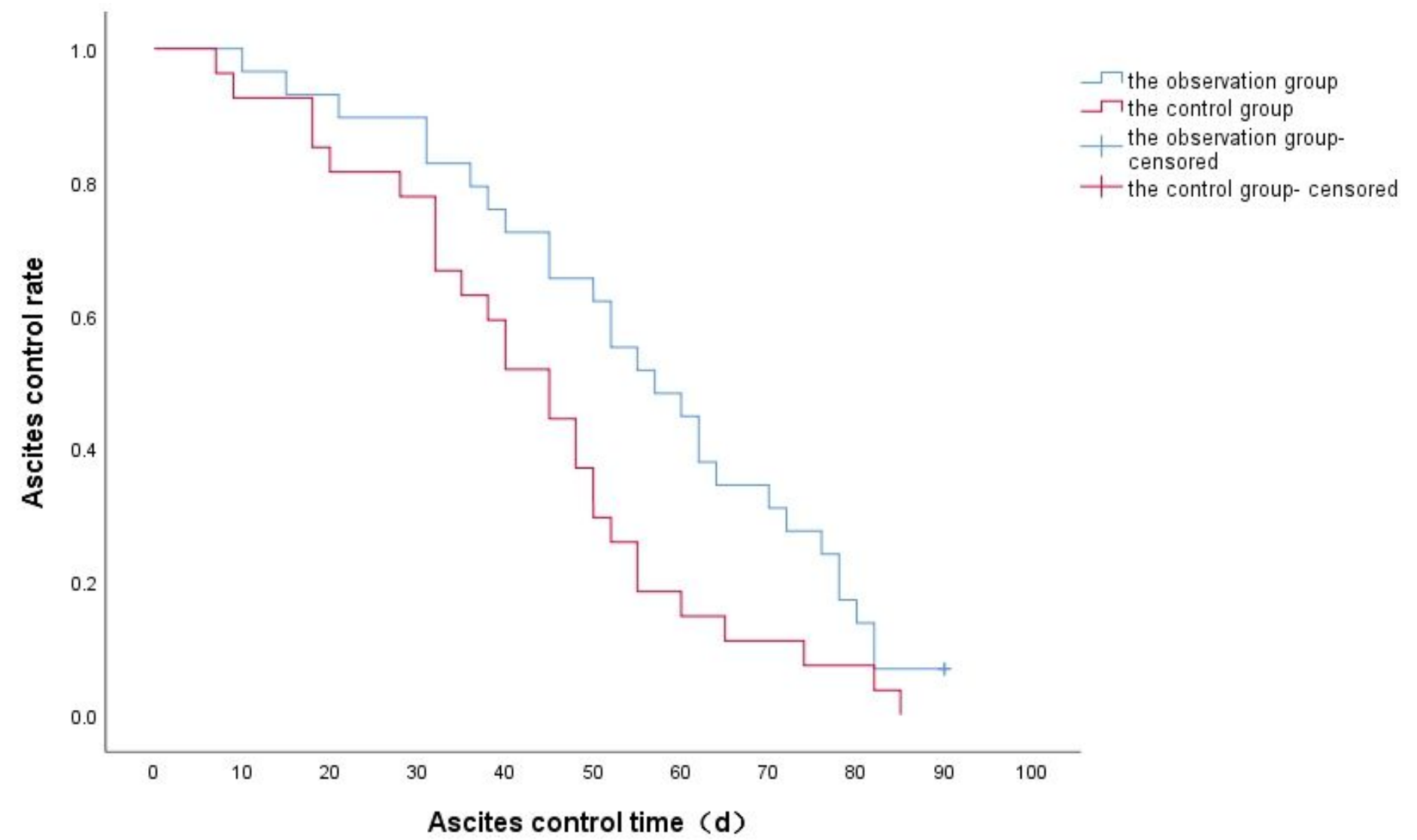

Figure 1

Ascites control time『Kaplan-Meier curve区

\section{Supplementary Files}

This is a list of supplementary files associated with this preprint. Click to download.

- Table1.jpg

- Table2.jpg

- Table3.jpg

- Table4.jpg 